\title{
The effect of being in a tip-of-the-tongue state on subsequent items
}

\author{
Bennett L. Schwartz
}

Published online: 16 November 2010

(C) The Psychonomic Society 2010

\begin{abstract}
The tip-of-the-tongue state (TOT) is the feeling that an inaccessible item will be recalled. In the TOT induction paradigm, participants are given a list of general information questions or word definitions, and the participants indicate whether they are in a TOT for each item. The present study explored the effect that being in a TOT for one item $(N)$ has on the recall and the likelihood of a TOT for the subsequent item $(N+1)$. Three experiments were conducted. All three experiments showed that TOTs do not affect the rate of recall for the next item but decrease the likelihood of a TOT for the next item. This effect extended to items occurring two items after the initial TOT $(N+2)$ in two experiments. Thus, TOTs are less likely to occur after another TOT than after an item not in a TOT. These data are interpreted within a metacognitive framework.
\end{abstract}

Keywords Tip-of-the-tongue states $\cdot$ Metacognition .

Retrieval

The tip-of-the-tongue state (TOT) is the feeling that a currently inaccessible item will be recalled (R. Brown \& McNeill, 1966; for reviews, see A. S. Brown, 1991, in press; Schwartz, 2006; Smith, 1994). When individuals experience TOTs, they feel confident that they can recall the item despite its current inaccessibility. TOTs have two important components; they are metacognitive experiences, and they are concerned with retrieval. Thus, TOTs have been considered as case studies of metacognition (e.g., Schwartz, 2006) and as windows on retrieval (e.g., A. S. Brown, 1991,

B. L. Schwartz $(\bowtie)$

Department of Psychology, Florida International University,

University Park,

Miami, FL 33199, USA

e-mail: bennett.schwartz@fiu.edu in press). TOTs have played an important role in a number of areas within cognitive psychology, including theories of memory, language production, and metacognition (see A. S. Brown, in press; Gollan \& Brown, 2006; Schwartz, 2006).

During TOTs, other cognitive processes may be interfered with or slowed down, particularly those that, like TOTs, originate in the prefrontal lobes (Maril, Simons, Weaver, \& Schacter, 2005; Schwartz, 2008). It is likely that being in a TOT draws attention away from other cognitive processes (Ryan, Petty, \& Wenzlaff, 1982). These cognitive processes may include retrieval of another item if, as in most experiments, the participant must answer a series of questions. That is, if a person is in a TOT for one item (e.g., the capital city of Uruguay), it may interfere with processing on a subsequent question (e.g., the capital city of Ukraine). Although some studies have examined the effect of TOTs on concurrent tasks, there is no literature on the effect of TOTs on subsequent items. Thus, this study represents an exploratory analysis of the effects of TOTs on subsequent items.

The present study examined the effect of TOTs and n-TOT states (those items not recalled for which people did not experience a TOT) on retrieval and TOTs for the next item of the list (item $N+1$ ). The study addresses two basic questions. First, does being in a TOT interfere with or facilitate retrieval of the $N+1$ item? Second, does being in a TOT interfere with or facilitate the likelihood of being in a TOT for an $N+$ 1 item? Because previous studies have shown that the presence of a TOT interferes with concurrent tasks (e.g., Ryan, Petty, \& Wenzlaff, 1982; Schwartz, 2008), it is likely that the presence of a TOT interferes with subsequent tasks, such as retrieval and TOTs for the next item.

Several studies have demonstrated that TOTs interfere with other cognitive processing, although all of this research is directed at concurrent tasks, rather than subsequent items. For example, Ryan et al. (1982) found 
that the presence of a TOT for a word definition interfered with performance on a number probe task. Participants were asked whether they were in TOTs for unretrieved word definitions. During this time period, they were also monitoring a string of digits for particular numerals. Ryan et al. found that accuracy and response time decreased in the number probe task when the participant was in a TOT for the word definitions, as compared with when the participant was in a don't know state. Similarly, Schwartz (2008) found that the presence of a TOT for general information questions interfered with performance on a digit span task. Schwartz (2008) also found that fewer TOTs occurred when participants had to maintain a series of digits in memory while retrieving targets than when they did not have this concurrent load. Thus, in each of these experiments, the presence of a TOT interfered with processing of an unrelated cognitive task.

Thus, being in a TOT demands attention that might otherwise be deployed elsewhere. Morris, Cleary, and Still (2008) found that feelings of familiarity-a type of judgment likely related to TOTs - are correlated with the allocation of attentional resources. The same may be true of TOTs. If so, being in a TOT may interfere with the processing of subsequent items. That is, the attentional resources that are allocated to retrieve a difficult item affect the retrieval behaviors for that item, leading to more resources being allocated to those items for which the participant is experiencing a TOT.

Assuming the $N+1$ is of average difficulty, it may not require additional resources to retrieve it, especially since divided attention has relatively little effect on retrieval (Naveh-Benjamin, Craik, Perretta, \& Tonev, 2000). Thus, the prediction is that TOTs on the $N$ item will not affect recall of the $N+1$ item. However, the prediction for TOTs is different. Because there may be a limited reservoir of metacognitive resources, the initial TOT may drain resources away from the $N+1$ item. This leads to the prediction that experiencing a TOT may interfere with the likelihood of a TOT on the $N+1$ item.

In the experiments described here, recall and TOTs were compared for $N+1$ items following TOTs and $N+1$ items following n-TOTs. In order to do so, data from three experiments were analyzed, two of which were originally conducted to examine different issues. Experiment 1a was originally intended to examine cross-language differences in TOTs. Experiment $1 \mathrm{~b}$ was originally intended to examine the effects of TOTs on the processing of other tasks (the Stroop effect). Experiment 1c was specifically designed for the research questions advanced in this article. In each experiment, analyses were performed concerning the likelihood of successful recall. If recall was not successful, I examined whether or not a TOT occurred for $N+1$ items immediately following a TOT. I then compared these results with the likelihood of recall and the likelihood of TOTs for $N+1$ items following n-TOTs. This comparison was important because it meant that any differences would be caused by the presence or absence of a TOT for the $N$ item, and not by whether it was recalled or not.

Therefore, the following hypotheses emerge from this reasoning. First, it is likely that a TOT for item $N$ will not affect retrieval of the $N+1$ item, because divided attention does not affect retrieval strongly. However, for TOTs, the attentional resources necessary to experience a TOT will affect a person's metacognition on the $N+1$ item. If, like familiarity (Morris et al., 2008), TOTs are associated with a call for more resources, there may be less monitoring capacity left when the $N+1$ item is presented to the participant. Therefore, the hypothesis is that being in a TOT will reduce the likelihood of being in a TOT for the $N+1$ item.

\section{Method}

Participants In Experiment 1a, the participants were 11 French-speaking students at Laval University who received $\$ 10$ Canadian for their participation. In Experiment 1b, the participants were 20 Florida International University students who received course credit for their participation. In Experiment $1 \mathrm{c}$, the participants were 43 Florida International University students who received course credit for their participation. Each participant was tested individually on a Macintosh computer during a session that lasted approximately $1 \mathrm{hr}$.

Materials Superlab4 software was used to run the experiments and collect the data. In Experiment 1a, the stimuli were 40 general information questions, presented in French. The stimuli were adapted from Bacon, Schwartz, PaireFicout, and Izaute (2007). Some questions were dropped from the Bacon et al. stimuli because they concerned aspects of French culture about which Quebeçois would not be familiar. Presentation order was randomized for each participant in Experiment 1a. In Experiments $1 \mathrm{~b}$ and 1c, the stimuli were 79 general information questions taken from the Nelson-Narens norms (Nelson \& Narens, 1980). In Experiments $1 \mathrm{~b}$ and $1 \mathrm{c}$, the order of presentation of general information questions was randomized for each participant.

Procedure Participants were first given detailed instructions about the procedure. They were told that they would be answering a series of general information questions. They were given an explanation of the meaning of the term tip of the tongue. TOTs were defined as "means that you feel that you know the target answer and will recall it soon." In Experiment 1a, a native Quebeçois French speaker gave the instructions. Instructions in French were 
adapted from Bacon et al.. Participants were encouraged not to guess and to indicate that they did not know, rather than to guess on any particular trial. Participants were instructed that they could pace themselves.

The experimenter then started the computer program that ran the experiment. The program randomized the order of questions for each participant. Each question appeared in the middle of the screen, and a prompt appeared beneath the question. If the participant decided to answer the question, he or she said the answer aloud, and the experimenter typed it in. Having the experimenter type in answers prevented spelling errors from confounding the pool of unrecalled items. If the participant gave the correct response, he or she moved on to the next question.

If the participant indicated that he or she did not know the correct answer (i.e., an omission error), the computer program asked the participant if he or she was experiencing a TOT. Incorrect answers (i.e., errors of commission) were not followed by TOT judgments. The experimenter typed in a "Y" if the participant was experiencing a TOT and an "N" if not. In Experiment $1 \mathrm{~b}$, the participants engaged in a Stroop task after making a TOT judgment, but before they made recognition judgments. The Stroop data will not be considered here. In Experiment 1a, the participant attempted recall of the first letter of the missing target after making a TOT judgment. First-letter data will not be considered here. In Experiment 1c, the participant moved directly to the recognition test.

Participants were then given a recognition test for the questions in all three experiments. They were shown the question, followed by four alternatives, one of which was the correct answer (all the questions in Experiments $1 \mathrm{~b}$ and 1c were from Wilkinson \& Nelson, 1984; those in Experiment 1a were from Bacon et al., 2007). A number accompanied each alternative. Participants spoke the number associated with the answer that they thought was correct, and the experimenter typed it in. The recognition distractors were all close associates or potentially correct answers. For example, for the question, "What was the last name of the first person on the moon?" all of the distractors were the names of other astronauts from the same time period. Following the recognition phase, the participants moved on to the next recall question.

\section{Results}

Procedures for Analysis

Statistical reliability was measured at $\underline{p}<.05$. Analyses were done with the presence or absence of a TOT as a quasiindependent variable with two levels (TOT and n-TOT).

Recall and recognition The means for recall, commission errors, and recognition are given in Table 1. Errors of commission were discouraged; participants were instructed not to guess if they were not sure of the correct answer. As such, commission error rates were low $(6 \%$ across experiments). Recognition judgments were made ony for unrecalled targets and were not made on errors of commission. Thus, the recognition data in Table 1 reflect only those items for which TOT judgments were made. Recognition performance was above chance $(25 \%)$ in all three experiments $($ Experiment 1a, $\underline{t}(10)=9.2$; Experiment $1 \mathrm{~b}, \underline{t}(19)=$ 18.5; Experiment 1c, $\underline{t}(42)=18.0)$.

The presence or absence of a TOT for the $N$ item had no effect on recall of $N+1$ items $(\underline{F}<1$ for all the experiments). That is, if a person had a TOT on the $N$ item, the person was just as likely to recall the $N+1$ item as when there was no TOT for the first item. Thus, the attention, if any, diverted toward TOTs does not interfere with recall of subsequent items. I also examined whether the presence of a TOT would affect performance on the $N+2$ item - that is, the item that occurred two items away from the initial TOT or n-TOT. The presence or absence of a TOT for the $N$ item had no effect on the recall of $N+2$ items $(F \mathrm{~s}<1)$.

The presence or absence of a TOT for the $N$ item had no effect on the rate of errors of commission for $N+1$ items. Because commission error rates were low, the analysis was combined across the three experiments but yielded nothing significant $(\underline{F}<1)$. There was no effect of a TOT on

Table 1 Recall, errors of commission, and recognition*

\begin{tabular}{|c|c|c|c|c|c|}
\hline \multirow[t]{2}{*}{ Study } & \multicolumn{2}{|l|}{ Recall } & \multicolumn{2}{|c|}{ Errors of Commission } & \multirow[t]{2}{*}{ Recognition } \\
\hline & Post TOTs & Post $n$-TOTs & Post TOTs & Post $n$-TOTs & \\
\hline Experiment 1a & $31 \%$ & $31 \%$ & $5 \%$ & $5 \%$ & $51 \%$ \\
\hline Experiment $1 \mathrm{~b}$ & $34 \%$ & $36 \%$ & $7 \%$ & $6 \%$ & $51 \%$ \\
\hline Experiment $1 \mathrm{c}$ & $37 \%$ & $38 \%$ & $6 \%$ & $7 \%$ & $69 \%$ \\
\hline
\end{tabular}

*Recognition $=$ percent correctly recognized of items originally not recalled 
commission errors for the $N+2$ items $(\underline{F}<1)$ across all experiments.

TOTs TOT rates were determined by examining the number of TOTs divided by the total number of unanswered questions (see Schwartz, 2006). TOTs following previous TOTs were determined by dividing the number of $N+1$ TOTs by the total number of TOT and n-TOT responses that directly follow a TOT response. TOTs following previous n-TOTs were determined by examining the number of TOTs on $N+1$ items divided by the total number of TOT and n-TOT responses that directly follow a $N$-TOT response. TOT rates for $N+1$ items are given in Table 2 for each of the three experiments. In all three experiments, there were fewer $N+1$ item TOTs after $N$ item TOTs than after $N$-item n-TOTs. That is, the presence of a TOT interfered with the experience of a TOT on the subsequent item. In Experiment 1a, the difference was significant, $\underline{F}(1,10)=14.85, M S E=.02$. In Experiment 1b, the difference was significant, $\underline{F}(1,19)=42.59, M S E=03$. In Experiment 1c, the difference was significant, $\underline{F}(1,41)=$ 8.51, MSE $=.04$.

Would the decrease in TOT rates persist beyond the subsequent item? In other words, would the interference from an $N$-item TOT continue to the item after the $N+1$ item? For Experiment 1c, an analysis examined whether the likelihood of a TOT interfered with TOTs occurring two items later (i.e., the $N+2$ item). TOT rates on $N+2$ items were still lower for targets after an initial TOT than they were for $N+2$ items after an n-TOT, $\underline{F}(1,41)=16.5, M S E=.04$. In fact, the means were statistically the same as immediately after the TOT $(27 \%$ for immediately after, $25 \%$ for the item after that; $\underline{F}<1)$. However, for the next item $(N+3)$-that is, three items after an initial TOT $(n+3)$-there was no difference between the TOT rate after n-TOTs and the TOT rate for that item, $\underline{F}<1$. This outcome is shown in Fig. 1.

This effect was also present in Experiment 1a, $\underline{F}(1,10)=$ $12.4, M S E=.03(M=25 \%$ for the $N+2$ item). However, the effect was not significantly significant for Experiment 2a, $\underline{F}(1,19)=2.05, M S E=04(M=27 \%)$. Fig. 1 shows only the data for Experiment $1 \mathrm{c}$, in which there was no

Table 2 TOTs contingent upon previous item

\begin{tabular}{lll}
\hline Study & TOT following TOTs* & TOTs following $n$-TOT** \\
\hline Experiment 1a & $23 \%$ & $50 \%$ \\
Experiment 1b & $15 \%$ & $30 \%$ \\
Experiment 1c & $27 \%$ & $39 \%$ \\
\hline
\end{tabular}

*Percentage of $N+1$ TOTs on items following unrecalled items given TOTs on $N$ items. **Percentage of $N+1$ TOTs on items following unrecalled items given $n$-TOTs on $N$ items

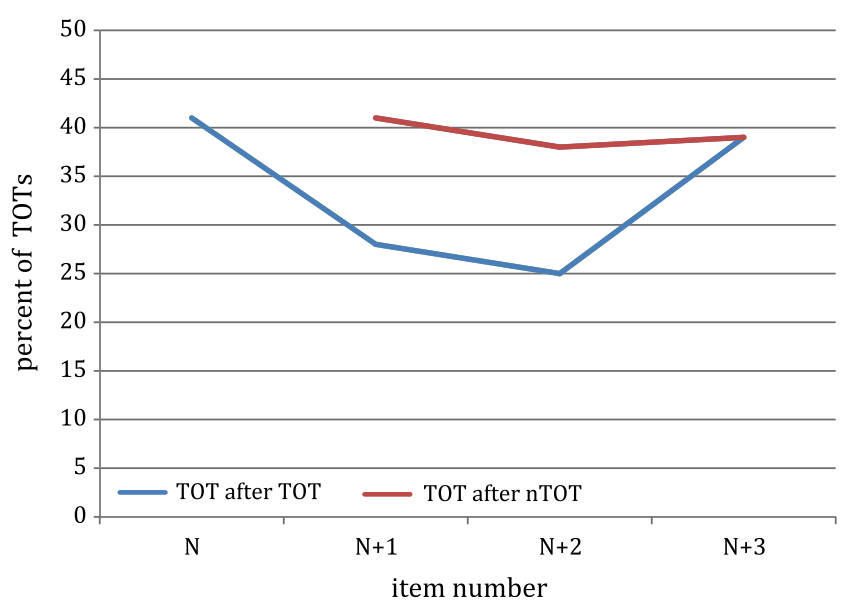

Fig. 1 The $y$-axis represents the proportion of TOTs experienced in Experiment $1 \mathrm{c}$ as a function of the items relation to an initial TOT. $N$ is the initial item - in either a TOT or an $n$-TOT state. $N+1, N+2$, and $N+3$ represent the items subsequent to that item, in order. The proportion of TOTs at $N$ includes all TOTs, whereas $N+1, N+2$, and $N+3$ represent only the TOT rates for those positions

intermediate task other than the recognition test between one item and the next.

In all three experiments, TOTs were better predictors of recognition than were n-TOTs, consistent with the vast majority of TOT research. Because the interest was not in TOT accuracy, accuracy will not be pursued further in this article.

\section{Discussion}

The primary finding is that participants experienced fewer TOTs for $N+1$ questions that followed TOTs than for those that followed n-TOTs. This occurred even though the presence or absence of a TOT had no effect on the likelihood of recall of the $N+1$ item. The study showed this decrease in TOTs across three experiments, two of which were in English and one of which was in French.

One explanation of the present data is that being in a TOT requires the allocation of attentional resources. Indeed, participants may be still attempting retrieval of the $N$ item when they have already been moved onto the $N+1$ item by the experimenter. The continued attention to the $N$ item does not interfere with the retrieval of $N+1$ items because divided attention does not affect retrieval as much as it does other processes, such as encoding (Naveh-Benjamin et al., 2000). Therefore, if we assume that there is a limited reservoir of metacognitive resources, because attentional resources are still focused on the $N$ item, there is little left over to elicit a TOT on the $N+1$ items. This accounts for the lower rate of TOTs following previous TOTs.

The present findings are consistent with the metacognitive approach to TOTs. In the metacognitive view, a TOT is 
a judgment made about the likelihood of recalling a currently inaccessible target item (Brennen, Vikan, \& Dybdahl, 2007; Cleary, 2006; Schwartz, 2008, 2010). That is, retrieval failure occurs because of a problem with some aspect of the retrieval process, but a metacognitive process that monitors retrieval produces the awareness of that problem. The TOT is the metacognitive awareness that retrieval is broken or slowed down.

As a metacognitive judgment, TOTs require sustained attention and the allocation of cognitive resources (Schwartz, 2008). This allocation of resources interferes with the ability to make similar metacognitive judgments while attention is diverted to the first TOT. However, because the act of retrieval is different from the act of making a metacognitive judgment, the allocation of resources does not interfere with recall, but only with the subsequent metacognitive judgment. In this way, the metacognitive view explains why TOTs interfere only with subsequent TOTs without interfering with subsequent recall.

That TOTs require attention and cognitive resources is consistent with a number of findings. First, TOTs interfere with performance on tasks that demand attentional resources, such as number probe tasks (Ryan et al., 1982) and verbal working memory (Schwartz, 2008). Second, the data are consistent with those in other studies examining memory experiences, which also show the relation between subjective experience and resource allocation. Morris et al. (2008) found that feelings of familiarity, an experience similar to the TOT (see Cleary, 2006), were associated with cognitive resource allocation. Third, recent data show that TOTs originate in the prefrontal lobes — the source of metamemory-rather than in areas of the brain associated with memory retrieval (Maril et al., 2005; see also Shimamura, 2008). Thus, this study is consistent with the generalization that TOTs are frontally based metacognitive monitoring judgments. As such, other frontally based judgments will interfere with them. In the present study, TOTs interfered with the likelihood of a TOT for the next item.

An alternative view of the present data postulates that the decrease in TOT rates was caused by demand characteristics (see Widner, Smith, \& Graziano, 1996). In this view, participants avoid reporting TOTs immediately after TOTs because they may not think the experimenter wants them to report so many TOTs. For example, Widner et al. showed that when participants thought items would be easy, they reported more TOTs than when they thought the items were difficult, even though all the participants received identical sets of items. This view, however, is unlikely to explain our data here. For Experiment 1c, I divided the participants into those who reported less than the median number of TOTs and those who reported more than the median number of TOTs. Both groups were less likely to report a TOT after a previous TOT. It is likely that those participants above the median had very low thresholds for reporting TOTs but did show a decrease in TOTs after previous ones.

Some researchers consider TOTs to originate from the same processes that produce retrieval, often called the direct access approach (e.g., Galdo-Alvarez, Lindin, \& Diaz, 2009; Gollan \& Brown, 2006). In this view, experimental variables should affect recall and TOTs in an equivalent manner. That is, if a variable inhibits the process of retrieval, it should both lower the rate of correct recall and lower the likelihood of a TOT. Of course, there are variables that do this (e.g., Gollan, Montoya, \& Bonanni, 2005). However, this view cannot explain dissociations between recall and TOT rates, as seen in the present study. Similarly, models based on inhibition of retrieval (e.g., Anderson, 2003) also would argue that recall would be inhibited as well as the TOTs.

The present findings contribute to a growing understanding of TOTs as metacognitive experiences that occur during some failed retrievals. The contention here is that there is mounting evidence to support the idea that TOTs are a nonconscious attribution based on clues provided during a failed retrieval (Cleary, Konkel, Nomi, \& McCabe, 2010; Metcalfe, Schwartz, \& Joaquim, 1993). Because of this, variables that interfere with the monitoring processes necessary to make this attribution will affect the rate of TOTs even if they do not affect retrieval (e.g., Schwartz, 2010). In the present study, the presence of a TOT for one item lowers the likelihood that subsequent items (up to $N+2$ ) will also be a TOT. This finding adds to our growing understanding of the TOT phenomenon.

\section{References}

Anderson, M. C. (2003). Rethinking interference theory: Executive control and the mechanisms of forgetting. Journal of Memory and Language, 49, 415-445.

Bacon, E., Schwartz, B. L., Paire-Ficout, L., \& Izaute, M. (2007). Dissociation between the cognitive process and the phenomenological experience of the TOT: Effect of the anxiolytic drug lorazepam on TOT states. Cognition and Consciousness, 16, 360-373.

Brennen, T., Vikan, R., \& Dybdahl, R. (2007). Are tip-of-the-tongue states universal? Evidence from an unwritten language. Memory, 15, 167-176. doi:10.1080/09658210601164743

Brown, A. S. (1991). A review of the tip-of-the-tongue experience. Psychological Bulletin, 109, 204-223. doi:10.1037/00332909.109.2.204

Brown, A. S. (in press). Tip of the tongue states. Psychology Press.

Brown, R., \& McNeill, D. (1966). The "Tip of the Tongue" Phenomenon. Journal of Verbal Learning and Behavior, 5, 325-337. doi:10.1016/S0022-5371(66)80040-3

Cleary, A. M. (2006). Relating familiarity-based recognition and the tipof-the-tongue phenomenon: Detecting a word's recency in the absence of access to the word. Memory \& Cognition, 34, 804-816.

Cleary, A. M., Konkel, K. E., Nomi, J. S., \& McCabe, D. P. (2010). Odor recognition without identification. Memory \& Cognition, 38, 452-460. 
Galdo-Alvarez, S., Lindin, M., \& Diaz, F. (2009). The effect of age on event-related potentials (ERP) associated with face naming and the tip-of-the-tongue (TOT) state. Biological Psychology, 81, 1423. doi:10.1016/j.biopsycho.2009.01.002

Gollan, T. H., Bonanni, M. P., \& Montoya, R. I. (2005). Proper names get stuck on bilingual and monolingual speakers' tip of the tongue equally often. Neuropsychology, 19, 278-287.

Gollan, T. H., \& Brown, A. S. (2006). From tip-of-the-tongue (TOT) data to theoretical implications in two steps: When more TOTs means better retrieval. Journal of Experimental Psychology: General, 135, 462-483. doi:10.1037/0096-3445.135.3.462

Maril, A., Simons, J. S., Weaver, J. J., \& Schacter, D. L. (2005). Graded recall success: An event-related fMRI comparison of tip of the tongue and feeling of knowing. NeuroImage, 24, 1130-1138.

Metcalfe, J., Schwartz, B. L., \& Joaquim, S. G. (1993). The cue familiarity heuristic in metacognition. Journal of Experimental Psychology: Learning, Memory, and Cognition, 19, 851-861. doi:10.1037/0278-7393.19.4.851

Morris, A. L., Cleary, A. M., \& Still, M. S. (2008). The role of autonomic arousal in feelings of familiarity. Consciousness and Cognition, 17, 1378-1385.

Naveh-Benjamin, M., Craik, F. I. M., Perretta, J. G., \& Tonev, S. T. (2000). The effects of divided attention on encoding and retrieval: The resiliency of retrieval processes. Quarterly Journal of Experimental Psychology, 53A, 609-625.

Nelson, T. O., \& Narens, L. (1980). Norms of 300 general-information questions: Accuracy of recall, latency of recall, and feeling-ofknowing ratings. Journal of Verbal Learning \& Verbal Behavior, 19, 338-368. doi:10.1016/S0022-5371(80)90266-2

Ryan, M. P., Petty, C. R., \& Wenzlaff, R. M. (1982). Motivated remembering efforts during tip-of-the-tongue states. Acta Psychologica, 51, 137-147.

Schwartz, B. L. (2002). Tip-of-the-tongue states: Phenomenology, mechanism, and lexical retrieval. Mahwah, NJ: Erlbaum.
Schwartz, B. L. (2006). Tip-of-the-tongue states as metacognition. Metacognition and Learning, 1, 149-158.

Schwartz, B. L. (2008). Working memory load differentially affects tipof-the-tongue states and feeling-of-knowing judgment. Memory \& Cognition, 36, 9-19. doi:10.3758/MC.36.1.9

Schwartz, B. L. (2010). The effects of emotion on tip-of-the-tongue states. Psychonomic Bulletin \& Review, 17, 82-87.

Shimamura, A. P. (2008). A neurocognitive approach to metacognitive monitoring and control. In J. Dunlosky \& R. A. Bjork (Eds.), Handbook of memory and metamemory: Essays in honor of Thomas O. Nelson (pp. 373-New York). New York: Psychology Press.

Smith, S. M. (1994). Frustrated feelings of imminent recall: On the tip-of-the tongue. In J. Metcalfe \& A. P. Shimamura (Eds.), Metacognition: Knowing about knowing (pp. 27-46). Cambridge, MA: MIT Press.

Widner, R. L., Smith, S. M., \& Graziano, W. G. (1996). The effects of demand characteristics on the reporting of tip-of-the-tongue and feelingof-knowing states. American Journal of Psychology, 109, 525-538

Wilkinson, T. S., \& Nelson, T. O. (1984). FACTRETRIEVAL2: A Pascal program for assessing someone's recall of generalinformation facts, confidence about recall correctness, feelingof-knowing judgments for nonrecalled facts, and recognition of nonrecalled facts. Behavior Research Methods, Instruments, and Computers, 16, 486-488.

The research was approved by the FIU Internal Review Board. The author thanks Ana-Sofia Navarrete for ideas and discussion, leading to this paper. The author thanks Lien Hernandez and Justine Tremblay for their assistance with data collection. The author thanks Sebastian Tremblay for allowing us to collect data at Laval University, Quebec, PQ. The author thanks two anonymous reviewers for their insight and ideas. 